Journal of

Cardiology and Vascular Medicine

\title{
A First in Class Treatment for Thrombosis Prevention. A Phase I study with CS1, a New Controlled Release Formulation of Sodium Valproate
}

\section{Niklas Bergh ${ }^{12^{*}}$, Jan-Peter Idström ${ }^{2}$, Henri Hansson ${ }^{3}$, Jonas Faijerson-Säljö ${ }^{2}$, Björn Dahlöf ${ }^{1,2}$}

${ }^{1}$ Department of Molecular and Clinical Medicine, Institute of Medicine, Sahlgrenska Academy, University of Gothenburg, Gothenburg, Sweden

${ }^{2}$ Cereno Scientific AB, Gothenburg, Sweden

${ }^{3}$ Galenica AB, Malmö, Sweden

${ }^{\star}$ Corresponding author: Niklas Bergh, The Wallenberg Laboratory for Cardiovascular Research Sahlgrenska University Hospital Bruna Stråket 16, 41345 Göteborg, Tel: +46 31 3421000; E-Mail: niklas.bergh@gu.se

Received Date: June 11, 2019 Accepted Date: July 25, 2019 Published Date: July 27, 2019

Citation: Niklas Bergh (2019) A First in Class Treatment for Thrombosis Prevention? A Phase I Study With Cs1, a New Controlled Release Formulation of Sodium Valproate. J Cardio Vasc Med 5: 1-12.

\begin{abstract}
Several lines of evidence indicate that improving fibrinolysis by valproic acid may be a fruitful strategy for thrombosis prevention. This study investigated the safety, pharmacokinetics, and effect on biomarkers for thrombosis of CS1, a new advanced controlled release formulation of sodium valproate designed to produce optimum valproic acid concentrations during the early morning hours, when concentrations of plasminogen activator inhibitor (PAI)-1 and the risk of thrombotic events is highest. Healthy volunteers $(n=17)$ aged 40-65 years were randomized to receive single doses of one of three formulations of CS1 (FI, FII, and FIII). The CS1 FII formulation showed the most favorable pharmacokinetics and was chosen for multiple dosing. Thirteen healthy volunteers received CS1 FII $276 \mathrm{mg}$ once daily for 14 days, and $552 \mathrm{mg}$ for a further 14 days. The mean (SD) peak plasma concentration (Cmax) of valproic acid at day 28 was $312.5(70.0) \mu \mathrm{mol} / \mathrm{L}$, and the median time to Cmax was 12 hours (range 9-16 hours). CS1 was safe well tolerated. Plasma PAI-1 concentrations decreased significantly from baseline to the end of the 28-day treatment period. Peak valproic acid concentrations were attained slightly later than peak PAI-1 levels. There was a close association $\left(\mathrm{R}^{2}=0.65\right)$ between plasma PAI- 1 concentrations and clot lysis time. In conclusion, this study showed that CS1 was safe and well-tolerated, showed favorable pharmacokinetics, as well as a significant reduction in PAI-1 and evidence of improved fibrinolysis. Further studies are warranted to further characterize the safety, tolerability and antithrombotic (fibrinolytic activity) of CS1.
\end{abstract}

Keywords: fibrinolysis; tissue-Plasminogen Activator (t-PA); Plasminogen Activator Inhibitor-1 (PAI-1)

(C)2019 The Authors. Published by the JScholar under the terms of the Creative Commons Attribution License http://creativecommons.org/licenses/ by/3.0/, which permits unrestricted use, provided the original author and source are credited. 


\section{Introduction}

Despite significant advances in antithrombotic therapy in recent years, thromboembolic conditions such as myocardial infarction, ischemic stroke, venous thrombosis, and pulmonary embolism remain major causes of death and morbidity in developed countries [1]. The extent of thrombosis in an individual reflects a balance between coagulation and fibrinolytic activity in the blood vessels. All current antithrombotic therapies focus on inhibiting the coagulation cascade, platelet function, or both, but dosing is often suboptimal because of the need to prevent dosedependent bleeding complications [2]. Hence, there remains a need for novel strategies to prevent thrombotic disease without increasing the risk of bleeding. Several lines of evidence suggest that improving the endogenous fibrinolytic capacity may offer such a rational therapeutic strategy. Impaired fibrinolysis $[3,4]$, due to either decreased production and depleted storage of tissue plasminogen activator ( $t$-PA) or increased expression of the principal inhibitor of fibrinolysis, plasminogen activator inhibitor-1 (PAI-1), has been reported in large studies in patients with coronary heart disease (CHD) or cardiovascular risk factors such as hypertension, obesity and/or smoking [5-9].

Similarly, genetic mutations that result in the decreased t-PA expression are associated with an increased risk of arterial thrombosis [10-12], and impaired fibrinolysis has also been shown to be predictive of future cardiovascular events [13-15]. Enhancement of the endogenous fibrinolytic system, therefore, offer a more effective approach than currently available therapy to prevent thrombotic disease without increasing the risk of bleeding.

Valproic acid, which has been used for many years for the treatment of epilepsy, is an epigenetic modulator of t-PA expression [16-18]. This compound has been shown to up-regulate t-PA expression in cultured endothelial cells $[17,18]$, large animal model [19] and to reduce thrombus formation in vivo [20]. In healthy volunteers [21] and patients with atherosclerosis [22] valproic acid have been shown to increase t-PA as well as supress PAI-1. These findings are consistent with evidence from large pharmaco-epidemiological studies in patients with epilepsy prescribed valproic acid as an anticonvulsant drug. In these studies treatment with valproic acid was associated with significant reductions in the risk of myocardial infarction and stroke in patients with epilepsy, compared with both the general population and patients treated with other anticonvulsants [23-26]. Such findings suggest that enhancement of endogenous t-PA storage and release as well as reduction of PAI-1 levels by valproic acid may reduce the risk of thromboembolic events.

The present study investigated the safety, tolerability, pharmacokinetics, and effect on biomarkers for thrombosis of CS1, a new advanced controlled-release formulation of sodium valproate in healthy volunteers. The mini-tablets are dissolved in the intestine and the sodium valproate salt is converted to valproic acid in contact with water [27]. CS1 is designed to produce optimum valproic acid concentrations during the early morning hours when circulating PAI-1 concentrations and the risk of thrombotic events are highest $[28,29]$.

\section{Materials and Methods}

The study was a Phase I study conducted at Clinical Trial Consultants, Uppsala Akademiska Hospital, Uppsala, Sweden. The first participant recruited was on $6^{\text {th }}$ of October 2017 and the last follow-up $27^{\text {th }}$ of March 2018. The first objective was to evaluate the safety, tolerability and the pharmacokinetics of single doses of three different formulations of CS1 with different release properties, to select one for subsequent multiple dosing. The second objective was to investigate the safety, tolerability and pharmacokinetics of CS1 during multiple dosing. In addition, pharmacodynamic effects on PAI-1, clot lysis, plasma a2 -antiplasmin complex (PAP), fibrinogen, high-sensitivity C-reactive protein (hs-CRP), platelet function, fibrin D-dimer levels, and bleeding time were to be evaluated as exploratory objectives. The study was conducted in accordance with the principles of the Declaration of Helsinki and Good Clinical Practice and with local regulatory requirements. The protocol and other study documents were approved by the Medical Products Agency in Uppsala, Sweden, and by the Independent Ethics Committee in Uppsala. The study is registered with the European Clinical Trials Database (EudraCT: study no. 2017-002140-32) and at ClinicalTrials.gov: NCT03903302. We also confirm that all ongoing and related trials for this drug formulation are registered.

\section{Study population}

Healthy volunteers of either sex were eligible for inclusion in the study if they were aged 40-65 years inclusive, and had a body mass index (BMI) between 27 and $35 \mathrm{~kg} / \mathrm{m}^{2}$. Besides, participants in the multiple ascending dose part of the study were required to have PAI-1 levels of $15-80 \mu \mathrm{g} / \mathrm{L}$. Participants were not included if they had significant medical conditions or thrombocyte plasma concentration (TPK), activated partial thromboplastin time (APTT) or an international normalized ratio (INR) that were significantly outside the normal ranges. Current smokers or users of nicotine products, and participants with 
current or previous alcohol or drug abuse were also excluded. Male participants were required to use condoms for 3 months after dosing, to prevent the possible transmission of study drug to a partner. Female participants were required to be post-menopausal or to have undergone hysterectomy or tubal ligation. Written informed consent was obtained from all participants before enrolment.

\section{Study design}

The study consisted of a randomized single dosing part, followed by a repeated ascending dosing part with the formulation from the single-dose part that showed the most favorable pharmacokinetics. The two parts were separated by a washout period of at least 14 days; participants enrolled in the single-dose part were also eligible to enter the repeated dosing part. During the first part, participants were randomized 1:1:1 to receive a single dose of one of three different formulations of CS1, designated FI, FII, and FIII. The randomization list was prepared using SAS ${ }^{\circledast}$ Proc Plan, SAS ${ }^{\circledR}$ version 9.4 (SAS Institute, Cary, NC). CS1 was provided as capsules containing 20 or 21 coated mini-tablets containing $275 \mathrm{mg}$ (FI) or $276 \mathrm{mg}$ (FII and FIII) sodium valproate; the three formulations differed in release design (see below). Data from the first six participants were used to validate the pharmacokinetic sampling schedule, while data from all participants in the single-dose part were used to determine the best CS1 formulation for the second part of the study.

During the repeated dose part of the study, all participants received FII once daily (i.e., total daily dose $276 \mathrm{mg}$ sodium valproate), for 2 weeks, after which the dose was doubled (i.e., $552 \mathrm{mg}$ sodium valproate) for a further 2 weeks.

All doses were taken at $20.00 \pm 2$ hours, with $240 \mathrm{~mL}$ of water. Consumption of alcohol, xanthine- or taurine-containing drinks, and grapefruit or grapefruit-containing products was not permitted during the study. Concomitant medications were not permitted, except for medications considered necessary by the investigator, acetaminophen at daily doses of up to $2000 \mathrm{mg}$ for up to 3 consecutive days, and antihistamines or nasal decongestants without cortisone for up to 10 days.

\section{Drug formulation}

The CS1 drug formulations consist of coated mini-tablets contained in hydroxypropyl methylcellulose (HPMC) capsules. Each mini-tablet contains approximately $14 \mathrm{mg}$ sodium valproate together with fumaric acid, hydroxypropyl cellulose, silica colloidal anhydrous and magnesium stearate. The mini-tablets are dissolved in the intestine and the sodium valproate salt is converted to valproic acid in contact with water [27]. The mini-tablets have two (FI) or three (FII and FIII) functional coating layers, comprising different combinations of coating polymers (Kollicoat ${ }^{\circledR}$ IR and Kollicoat ${ }^{\oplus}$ SR 30 D from BASF, Eudragit ${ }^{\oplus}$ FS 30 D and Eudragit ${ }^{\oplus}$ L 30 D-55 from Evonik) and talc, silica colloidal anhydrous, glycerol monostearate and triethyl citrate as coating additives. The coated mini-tablets have been designed to create a specific profile of valproic acid release that matches the circadian changes in PAI-1 [29]. The properties of the coating were investigated using established in vitro dissolution techniques [30]. This set-up mimic the passage in the GI tract and give data on drug release.

\section{Pharmacokinetic analyses}

During both parts of the study, venous blood samples (approximately $4 \mathrm{~mL}$ ) for the measurement of valproic acid were obtained via an indwelling cannula within 5 minutes prior to dosing, immediately after dosing, and at 15, 30 and 45 minutes, $1,2,3,4,6,8,12,16,24,38,50$, and 74 hours after dosing. Plasma concentrations of valproic acid were measured using a particle-enhanced turbidimetric inhibition immunoassay (PETINIA) on an Abbott Architect c800 platform, with a lower limit of quantification (LOQ) of $50 \mu \mathrm{mol} / \mathrm{L}$. The following pharmacokinetic parameters were calculated by non-compartmental methods using Phoenix WinNonlin ${ }^{\circledast}$ software, version 7.0 or above ( Pharsight Corporation, St Louis, MO): maximum plasma concentration $(\mathrm{Cmax})$, time to $\mathrm{Cmax}(\mathrm{T} \max )$, area under the concentration-time curve from 0 to infinity (AUCinf), AUC from 0 to time of last measured concentration (AUClast), first-order terminal elimination rate constant $(\boldsymbol{\lambda})$, terminal half-life $(\mathrm{T} 1 / 2)$, apparent total clearance $(\mathrm{CL} / \mathrm{F})$, and apparent volume of distribution $(\mathrm{Vd} / \mathrm{F})$.

\section{Safety evaluation}

Safety and tolerability were evaluated throughout the study by a recording of adverse events (spontaneously reported and elicited by questioning), physical examination, recording of vital signs and 12-lead electrocardiogram (ECG), and clinical laboratory investigations (clinical chemistry, hematology, APTT, and INR).

\section{Pharmacodynamic evaluation}

Blood samples for measurement of pharmacodynamic 
variables were taken at the same time as those taken for pharmacokinetic analysis. PAI-1 concentrations, PAP, fibrinogen, hs-CRP, platelet function, fibrin D-dimer levels, and bleeding time were measured using routine analytical techniques. Also, inhibition of fibrinolysis was measured in a turbidimetric clot lysis assay system using citrated platelet-poor plasma [31]. In this assay, fibrin formation is started when the citrated plasma is re-calcified by the addition of calcium chloride, which leads to the endogenous formation of thrombin. t-PA is then added to activate plasminogen to plasmin, resulting in fragmentation of fibrin particles.

\section{Statistical analysis}

No formal sample size calculation was performed. It was considered that a sample size of $>30$ volunteers would be sufficient to achieve the aims of the study.

Pharmacokinetic analyses were conducted in the per-protocol (PP) population, which consisted of all participants who completed the study without protocol violations. Safety and tolerability were analyzed in the full analysis population, which included all participants who received at least one dose of CS1. All data (pharmacokinetic, safety and pharmacodynamic) were summarized using descriptive statistics. In the repeated dos- ing part of the study, the effects of low and high CS1 exposure on PAI-1 concentrations were compared using the Wilcoxon signed-rank test. The correlation between PAI-1 and the clot-lysis time was evaluated using least squares regression analysis. All statistical analyses were performed using SAS Version 9.4 software (SAS Institute, Cary, NC), and $P$ values below 0.05 were considered significant.

\section{Results}

During the single-dose part of the study, 17 participants were randomized to treatment with one of the three different CS1 formulations (FI, FII and FIII); all of these completed the study (Figure 1). In the multiple ascending dose part, 13 volunteers were enrolled, all of whom completed the study. Five participants were included in both parts of the study. All participants in the single-dose part were included in both the full analysis population and the PP population. In the repeated dosing part of the study, all 13 participants were included in the full analysis population, but one was excluded from the PP population due to a protocol violation (PAI-1 concentration outside the permitted range at screening). Demographic characteristics of all participants are summarized in Table 1. In general, the characteristics of participants in each part were similar, except for a higher proportion of females in the multiple dosing phase.

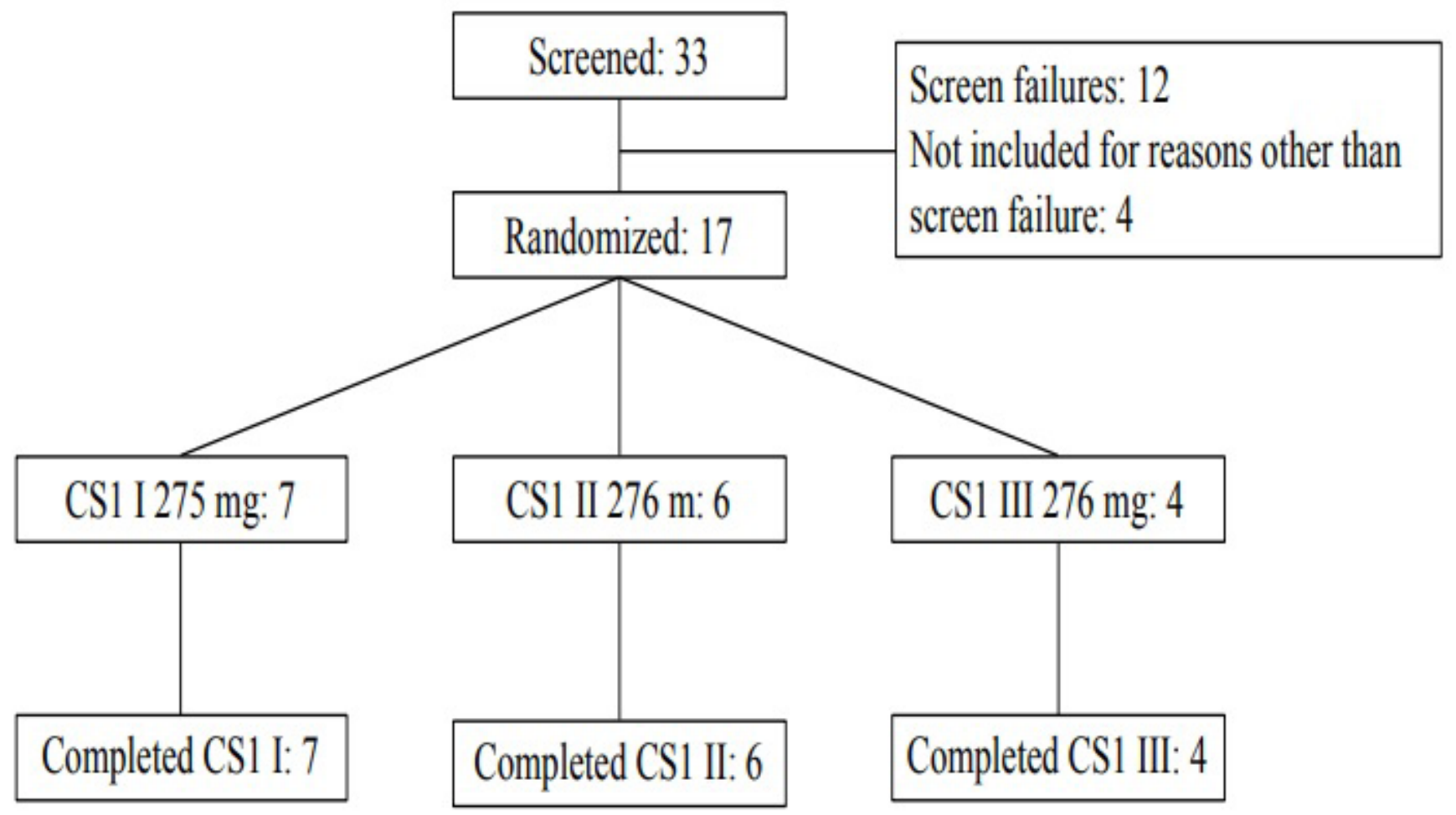

Figure 1. Study schematic with the disposition of participants in the single-dose part of the study. 


\begin{tabular}{|c|c|c|c|c|c|}
\hline & \multicolumn{4}{|c|}{$\begin{array}{l}\text { Single-dose }(n=17) \text { Three } \\
\text { formulations of CS1 }\end{array}$} & \multirow[t]{2}{*}{$\begin{array}{r}\text { Repeated } \\
\text { dosing } \\
(n=13)\end{array}$} \\
\hline & FI $(n=7)$ & FII $(n=6)$ & FIII $(n=4)$ & Total & \\
\hline Age (years) & $50.6(6.9)$ & $52.2(8.2)$ & $57.3(7.0)$ & $52.7(7.4)$ & $52.2(6.6)$ \\
\hline $\begin{array}{l}\text { Gender (female/ } \\
\text { male) }\end{array}$ & $\begin{array}{c}4 / 3 \\
(57 \% / 43 \%)\end{array}$ & $\begin{array}{c}3 / 3 \\
(50 \% / 50 \%)\end{array}$ & $\begin{array}{c}1 / 3 \\
(25 \% / 75 \%)\end{array}$ & $\begin{array}{c}8 / 9 \\
(47 \% / 53 \%)\end{array}$ & $\begin{array}{c}5 / 8 \\
(38 \% / 62 \%)\end{array}$ \\
\hline $\begin{array}{l}\text { Race (White/Hispan- } \\
\text { ic or Latino) }\end{array}$ & $\begin{array}{c}6 / 1 \\
(86 \% / 14 \%)\end{array}$ & $\begin{array}{c}5 / 1 \\
(83 \% / 17 \%)\end{array}$ & $\begin{array}{c}3 / 1 \\
(75 \% / 25 \%)\end{array}$ & $\begin{array}{c}14 / 3 \\
(82 \% / 18 \%)\end{array}$ & $13(100 \%)$ \\
\hline $\begin{array}{l}\text { Body mass index } \\
\left(\mathrm{kg} / \mathrm{m}^{2}\right)\end{array}$ & $29.7(2.2)$ & $33.3(1.2)$ & $30.8(2.8)$ & $31.2(2.5)$ & $30.9(2.7)$ \\
\hline Height $(\mathrm{cm})$ & $170(12.7)$ & $168(10.8)$ & $172(8.8)$ & $170(10.7)$ & $168(12.5)$ \\
\hline Weight (kg) & $86(12.1)$ & $94.2(14.4)$ & $90.3(10.4)$ & $89.9(12.4)$ & $87.2(12)$ \\
\hline
\end{tabular}

Table 1. Demographic characteristics of participants. Results are presented as mean (SD) or as number (\%).

\section{Pharmacokinetics of CS1}

\section{Single-dose part}

Following single dosing, the mean (SD) Cmax for FI and FII was $74.3(17.5) \mu \mathrm{mol} / \mathrm{L}$ and $76.1(56.4) \mu \mathrm{mol} / \mathrm{L}$, respectively, and the median (range) Tmax was 13.0 (10.1-18) hours and 14.0 (0-18) hours, respectively. Mean (SD) drug exposure,

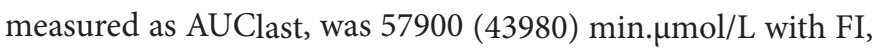
and 80470 (71260) min. $\mu \mathrm{mol} / \mathrm{L}$ with FII. Due to this higher exposure (higher relative bioavailability) with FII, compared with FI, this formulation was chosen for the multiple dosing part of the study. Due to the low number of participants receiving FIII $(n=4)$ and high variability, it was not possible to calculate pharmacokinetic parameters for this formulation.

\section{Multiple-dose part}

Plasma valproic acid concentrations during multiple dosing with CS1 (FII), 276 mg and 552 mg, are shown in Figure 2 , and the time course of valproic acid concentrations ( 0 to 74 hours post-dose) following the final dose on Day 28 is shown in Figure 3. With both doses, steady-state conditions were reached within 7 days. At the end of the dosing period, mean (SD) Cmax was $312.5(70.0) \mu \mathrm{mol} / \mathrm{L}$, and median Tmax was reached after 12.0 hours (range 9.0-16.0 hours) The mean (SD) terminal elimination rate constant $(\lambda)$ was 15.1 (4.2) hours), and the accumulation index calculated using this constant was 1.5 (0.2). Following the attainment of Cmax after the final dose, valproic acid concentrations declined to undetectable levels over approximately 3.5 days (Figure 3 ).

\section{Safety and tolerability}

During the single-dose part of the study, seven participants (41\%) reported a total of 12 adverse events, all of which were mild or moderate in intensity. A total of four adverse events in two participants were considered to be possibly or probably related to study medication: fatigue was reported by one participant receiving FI, and fatigue, headache, and hyperhidrosis were reported by one participant receiving FII.

In the multiple ascending dose part of the study (total 4 weeks dosing), 11 participants (85\%) reported a total of 46 adverse events CS1 (FII); seven participants reported 22 adverse events during treatment with the $256 \mathrm{mg}$ dose, and 10 reported 24 adverse events during treatment with the $552 \mathrm{mg}$ dose. All adverse events were rated as mild or moderate, and approximately two-thirds were considered to be possibly related to study medication; of these, the only events occurring in more than one participant were headache, fatigue, restless legs syndrome and dry mouth ( $\mathrm{n}=2$ each). No serious adverse events were reported during the study, and no participant withdrew from the study because of adverse events. No clinically significant changes in vital signs, ECG, physical examinations, bleeding events or clinical laboratory investigations were observed during any part of the study.

\section{Pharmacodynamics}

Changes in pharmacodynamic parameters during the multiple dosing part of the study are summarized in Table 2. Plasma PAI-1 concentrations did not change significantly during treatment with FII $276 \mathrm{mg}$ but decreased significantly during subsequent treatment with FII 552 mg (Figure 4). The mean (SD) 


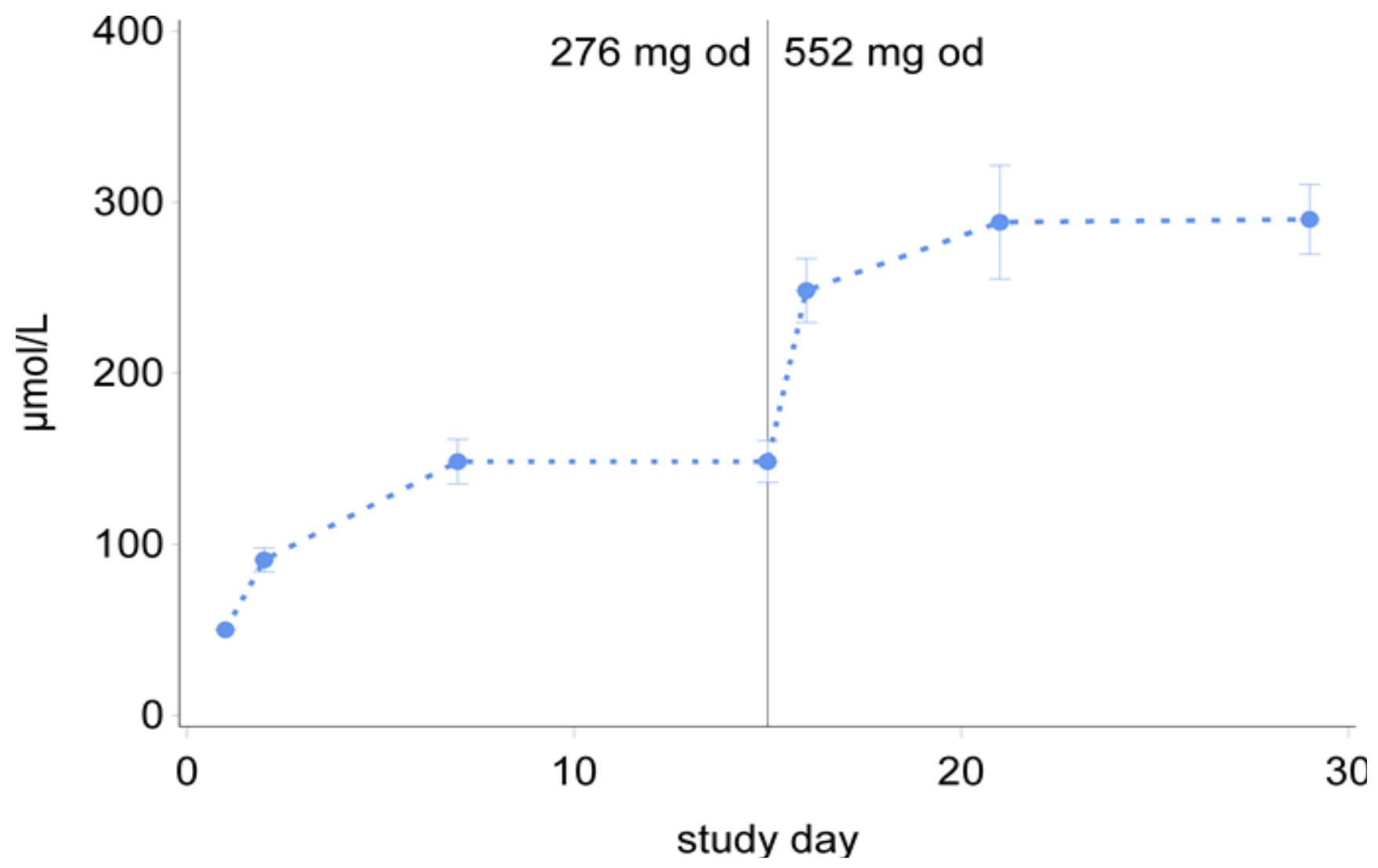

Figure 2. Mean (SEM) plasma valproic acid concentrations during daily dosing with CS1, FII 276 mg daily (Days 1-14) and 552 mg daily (Days 15-28).

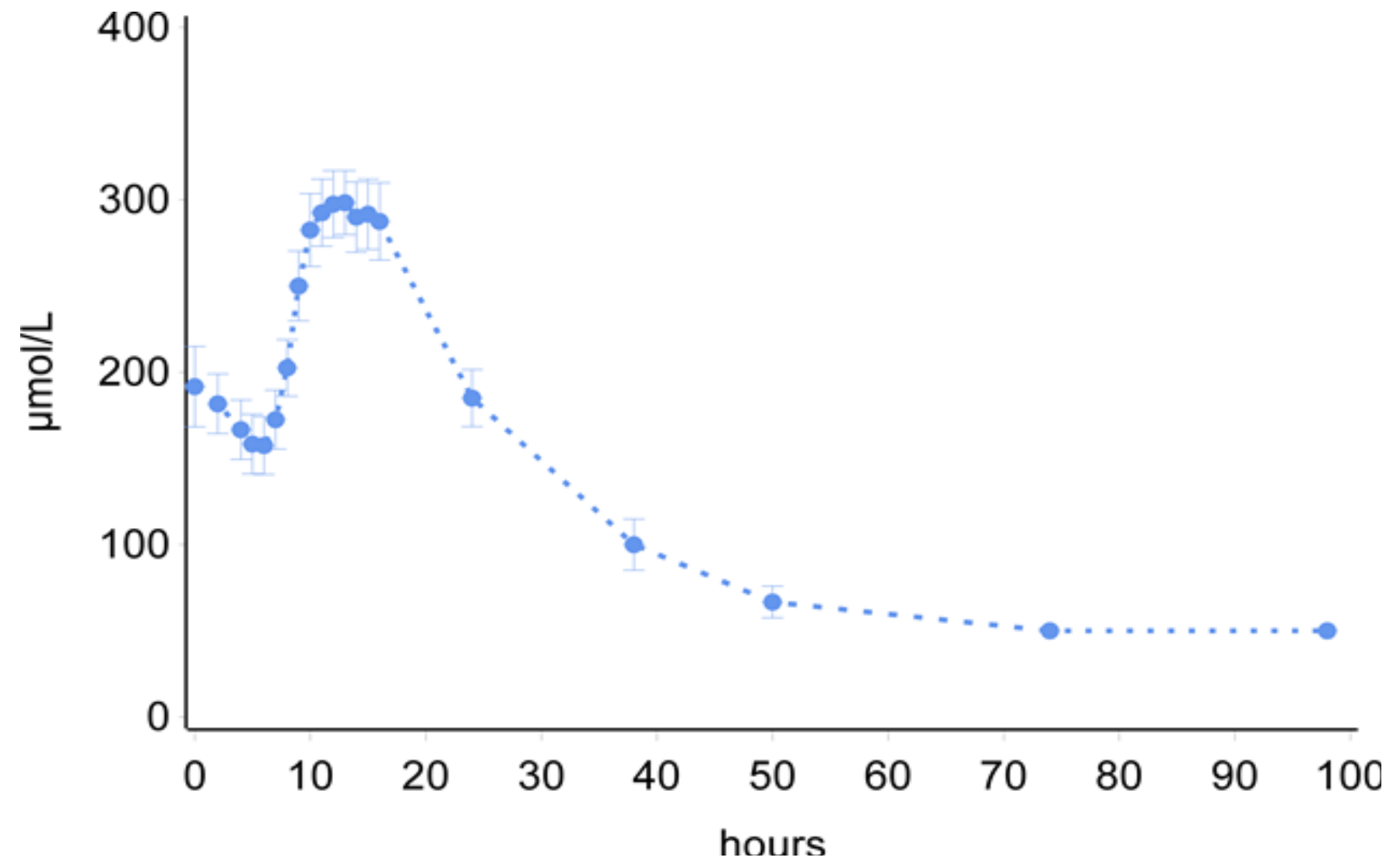

Figure 3. Mean (SEM) plasma valproic acid concentrations following the final dose on Day 28. 
Table 2. Changes in pharmacodynamic parameters from baseline (Day 1) to Days 15 (end of $276 \mathrm{mg}$ dosing) and 29 (end of $552 \mathrm{mg}$ dosing) of multiple dosing with CS1, FII. Results are presented as mean (SD).

\begin{tabular}{|l|l|l|l|}
\hline & Baseline & $\begin{array}{l}\text { Day 15 } \\
\text { Measured 14 hours after } \\
\text { last dose }\end{array}$ & $\begin{array}{l}\text { Day 29 } \\
\text { Measured 14 hours after } \\
\text { last dose }\end{array}$ \\
\hline PAI-1 $(\mu \mathrm{g} / \mathrm{L})$ & $52.3(28.6)$ & $62.2(37.5)$ & $41.2(22.5)^{*}$ \\
\hline PAP $(\mu \mathrm{g} / \mathrm{L})$ & $434.2(555.4)$ & $391.3(483.0)$ & $353.1(392.5)$ \\
\hline Fibrinogen $(\mathrm{g} / \mathrm{L})$ & $3.82(0.56)$ & $3.49(0.48)$ & $3.33(0.58)$ \\
\hline hs-CRP $(\mathrm{mg} / \mathrm{L})$ & $4.27(5.21)$ & $2.94(3.06)$ & $4.44(3.98)$ \\
\hline Platelets unit $\left(10^{9} / \mathrm{L}\right)$ & $161.7(55.4)$ & $148.4(32.4)$ & $155.7(40.0)$ \\
\hline D-dimer $(\mathrm{mg} / \mathrm{L})$ & $0.35(0.08)$ & $0.37(0.08)$ & $0.61(0.45)$ \\
\hline Bleeding time $($ minutes $)$ & $4.96(1.14)$ & $5.00(1.60)$ & $4.67(1.15)$ \\
\hline
\end{tabular}

hs-CRP: high-sensitivity C-reactive peptide; PAP: plasmin- a2-antiplasmin complex; PAI-1: plasminogen activator inhibitor ${ }^{*} P=0.0425$ versus Day 15, Wilcoxon signed rank test

reduction in plasma PAI-1 concentrations, measured 14 hours after the last dose of FII, between days 15 and 29 was -21.1 (32.6) $\mu \mathrm{g} / \mathrm{L}(P=0.0425$, Wilcoxon signed-rank test; Table 2$)$. Comparison of the plasma concentration-time profiles for valproic acid and PAI-1 over 24 hours after the last dose of FII showed that peak PAI-1 concentrations were attained slightly earlier than peak valproic acid concentrations (mean 10 hours versus 12 hours, respectively, Figure 4). Plasma PAP concentrations were reduced, compared with baseline, at all time points except Day 2. On day 29, the mean concentration measured 14 hours after dosing was 353 (393) ng/mL, compared with 434 (555) ng/mL at baseline (Table 2). Similar trends were seen in plasma fibrinogen concentrations; fibrinogen $\mathrm{D}$-dimer concentrations were increased from baseline levels at all time points during treatment with CS1(FII), but these increases were relatively small (Table 2). Mean bleeding time was similar to baseline at all time points during the entire dosing period, except for day 29, when the mean (SD) bleeding time was 4.7 (1.2) minutes, compared with 5.0 (1.1) minutes at baseline (Table 2).

Measurements of clot lysis time over 24 hours showed a close association between clot lysis time and plasma PAI-1 concentrations (Figure 5). Overall $(n=125)$, there was a curvilinear correlation $\left(\mathrm{R}^{2}=0.65\right)$ between clot lysis time and plasma PAI-1 concentrations (Figure 6). During the multiple-dose part of the study, clot lysis time was 446 minutes at baseline and 312 minutes on day 28 at $08.00(\mathrm{p}=0.30)$.

\section{Discussion}

This study investigated the safety, pharmacokinetics, and effect on biomarkers for thrombosis of CS1. CS1 is an advanced controlled-release formulation of sodium valproate, the sodium salt of valproic acid, and the formulation has been de- signed to release valproic acid during the night and early morning following administration in the evening. This will maximize the antithrombotic effect of valproic acid at the time when PAI-1 activity is at its highest plasma level [29] while minimizing the risk of symptomatic side-effects. The principle behind the CS1 controlled-release formulations, described above was to achieve a peak concentration of valproic acid at a median of 12 hours after dosing and a trough concentration during the day which is half of the peak concentrations during the night and early morning (i.e., a peak/trough ratio of 2; see Figure. 3).

With this specific pharmacokinetic profile, it is expected that drug concentration-related AEs will be minimized, whereas the antithrombotic efficacy should be enhanced. This is in contrast to the therapeutic use of valproic acid in the treatment of epilepsy, where a stable plasma concentration higher than the peak concentration in this study, over 24 hours is the target for successful treatment [32]. CS1 is designed to optimize the clinical efficacy of the endogenous fibrinolytic system and minimize the risk of bleeding. Indirect evidence that it is safe to stimulate the an endogenous fibrinolytic system without increased bleeding risk was demonstrated in a clinical study in patients with pulmonary emboli treated with the carboxypeptidase $\mathrm{U}$ (CPU) inhibitor (also known as active thrombin- activatable fibrinolysis inhibitor) AZD9684 [33]. CPU is an endogenous protein involved in the prevention of fibrinolysis, and inhibition of this protein may stimulate the breakdown of a thrombus. The risk of bleeding was not increased in this study. However, the development of AZD9684 was terminated due to the short halflife. Proof of concept for the effectiveness of stimulating the endogenous fibrinolytic system has also been shown in a thrombosis model in mice [20] Pretreatment with valproic acid reduced thrombus formation without any increased bleeding tendency. 


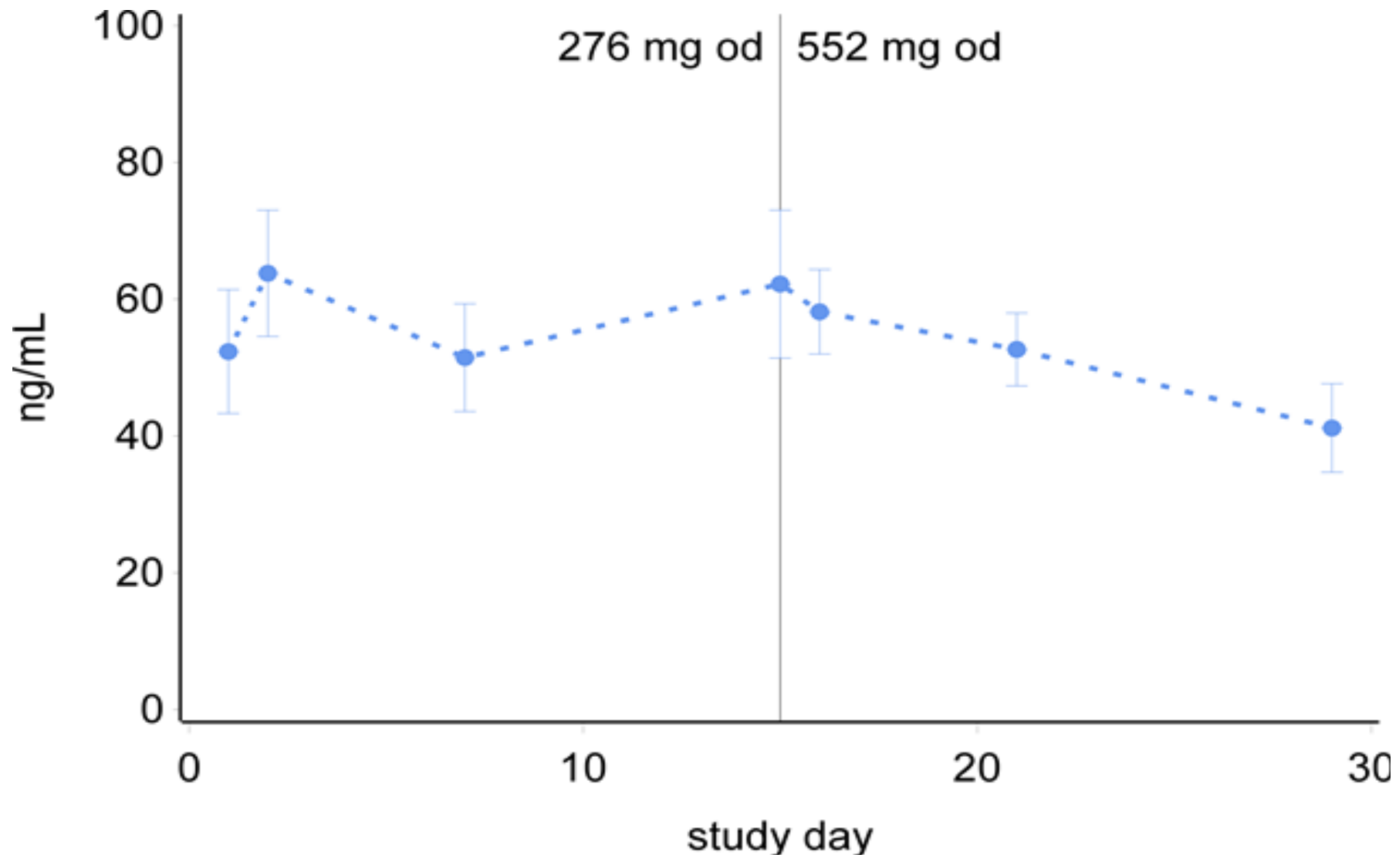

Figure 4. Mean (SEM) plasma concentrations of plasminogen activator inhibitor-1 (PAI-1) during treatment with CS1, FII, 276 $\mathrm{mg}$ daily (Days 1-14) and $552 \mathrm{mg}$ daily (Days 15-28) inthe multiple ascending dosing part of the study.

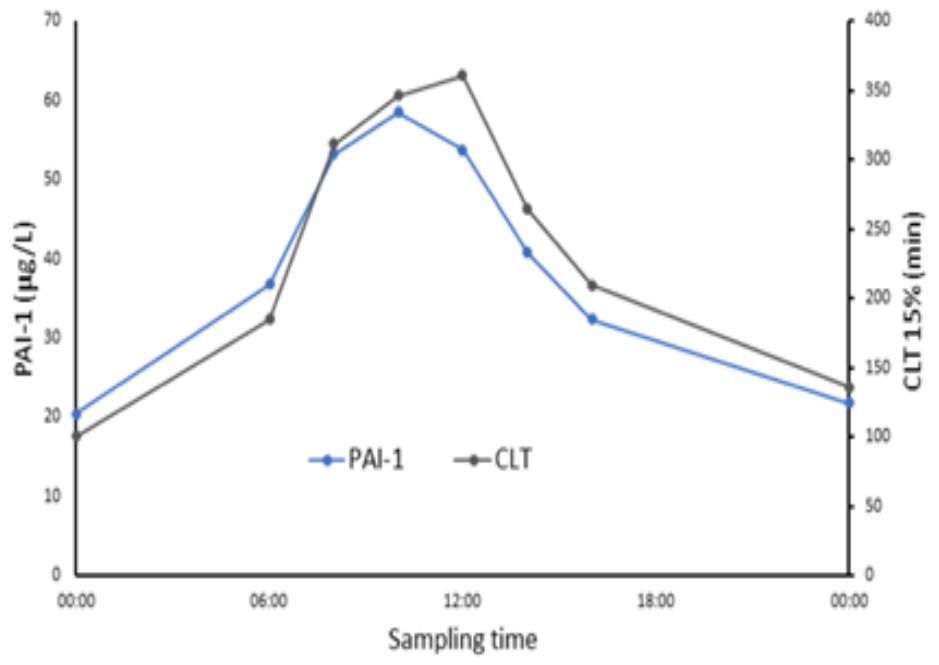

Figure 5. Association between clot lysis time and mean (SEM) plasma PAI-1 concentrations over 24 hours. 


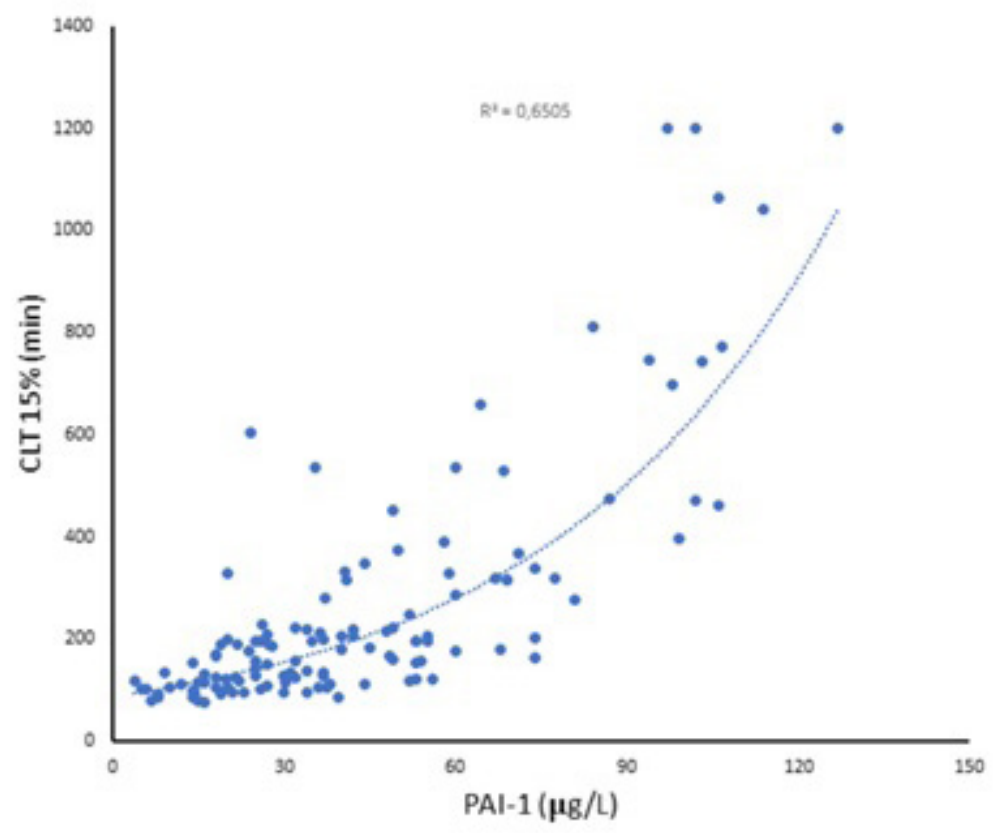

Figure 6. Overall correlation $(\mathrm{n}=125)$ between clot lysis time and plasma PAI-1 concentrations.

The present study was a proof of mechanism study in a man of the pharmacokinetics, pharmacodynamics, and safety of CS1 for the prevention of thromboembolic events. The results show that, during repeated dosing with $552 \mathrm{mg}$ CS1 as FII, given in the evening, peak plasma valproic acid concentrations are attained at approximately the same time as expected peak PAI-1 concentrations, which are known to be maximal during the early morning hours [29]. Furthermore, pharmacodynamic analyses suggest that, in addition to the well-documented stimulation of endothelial t-PA expression by valproic acid [17-20, 22], the 552 mg dose of this formulation also produces a significant reduction in PAI-1 concentrations.

Similar effects on PAI-1 have been obtained in two previous studies, one with healthy male volunteers treated for 2 weeks and one with patients with atherosclerosis treated for 4 weeks with a higher daily dose (500 mg twice daily) of a depot release formulation of valproic acid [21,22]. Together with evidence that valproic acid reduces thrombus formation in animal models [20], and that the clinical use of valproic acid in patients with epilepsy is associated with a reduced risk of thromboembolic events such as myocardial infarction and stroke [23-26], these findings suggest that valproic acid treatment offers a rational therapeutic strategy for the prevention of thrombotic disease.

The present study also showed a good correlation between clot lysis time and plasma PAI-1 concentrations. This is an important finding because clot lysis time may predict arte- rial and venous recurrent thromboembolic events [34-39]. It has been reported that patients with chronic atrial fibrillation and previous stroke have prolonged clot lysis [39]. Similarly, in a subanalysis of the Platelet Inhibition And Patient Outcomes (PLATO) trial, which compared ticagrelor with standard therapy in patients with acute myocardial infarction, in vitro fibrin clot formation and lysis assessed a few days after symptom onset were unaffected by ticagrelor, but clot lysis time was predictive of clinical outcome [40]. Furthermore, in in vitro studies, aspirin has been demonstrated to loosen fibrin networks and increase the thickness of fibrin fibers, leading to a $30 \%$ reduction in clot rigidity and enhanced clot lysis [41]. Clot lysis time is a useful method for the investigation of the different drugs effect on hemostasis in clinical settings [42].

The doses of CS1 used in this study are lower than those used in the treatment of epilepsy, where maintenance doses are usually 1-2 g daily [43]. Both doses were well tolerated: all adverse events were rated as mild or moderate in severity, and no treatment-related adverse events occurred in more than two participants. No participant withdrew from the study because of adverse events. In addition to the low dose of valproic acid, the use of a new advanced controlled-release formulation with peak concentrations occurring during the night might also be expected to enhance tolerability. Importantly, no adverse events related to serious bleeding were reported, suggesting that the fibrinolytic effect of valproic acid is not associated with an increased risk of such events. This is consistent with findings from previous studies in animals [20]. Although histone deacetylase inhibitors 
(HDACi ) have been reported to decrease platelet counts $[44,45]$ and inhibit platelet function [46], no such effects were observed in the present study.

Limitations of this study include the small sample size and the open design. Both of these are typical of Phase I studies; however, the sample size was sufficient to adequately characterize the pharmacokinetics of CS1. In summary, the new advanced controlled-release formulation of sodium valproate, CS1, was safe and well-tolerated and showed desirable pharmacokinetic properties.

Furthermore, this first clinical study with CS1 showed a significant reduction in PAI-1 and an indication of improved fibrinolysis. This, together with the previously documented increase in production, storage and release of t-PA following valproic acid treatment, gives support to the notion that CS1 could shift the t-PA/PAI-1 -balance in a favorable way at a dose well below traditional valproic acid use.

We conclude that further studies are warranted to determine whether these effects translate into clinically relevant antithrombotic activity in vivo, and to further characterize the safety and tolerability of CS1, a potentially first in the class alternative for the prevention of thrombosis events based on enhanced endogenous fibrinolysis.

\section{Acknowledgments}

Medical writing and editorial support in the preparation of this paper was provided by Dr. Michael Shaw (Anagram Communications Ltd, UK), and funded by Cereno Scientific AB (Gothenburg, Sweden).

\section{Declaration of Funding Source}

The study was funded by Cereno Scientific AB (Gothenburg, Sweden).

\section{References}

1. Day I.S.C.f.W.T (2014) Thrombosis: a major contributor to the global disease burden. Thromb Res134:931-938.

2. Capodanno D, et al. (2019) Management of Antithrombotic Therapy in Atrial Fibrillation Patients Undergoing PCI: JACC State-of-the-Art Review. J Am Coll Cardiol 74: 83-99.

3. Poli KA, et al. (2000) Association of blood pressure with fibrinolytic potential in the Framingham offspring population. Circulation101: 264-9.

4. Song C, et al. (2017) Causal Effect of Plasminogen Activator Inhibitor Type 1 on Coronary Heart Disease. J Am Heart Assoc 6.

5. Hrafnkelsdottir T, et al. (2004) Impaired endothelial release of tissue-type plasminogen activator in patients with chronic kidney disease and hypertension. Hypertension 44: 300304.

6. Hrafnkelsdottir T, et al. (1998) Impaired capacity for endogenous fibrinolysis in essential hypertension. Lancet 352: 1597-1598.

7. Newby DE, et al. (2001) Impaired coronary tissue plasminogen activator release is associated with coronary atherosclerosis and cigarette smoking: a direct link between endothelial dysfunction and atherothrombosis. Circulation 103: 1936-1941. 8. Osterlund B, et al. (2008) Impaired myocardial t-PA release in patients with coronary artery disease. Acta Anaesthesiol Scand 52: 1375-1384.

9. Van Guilder GP, et al. (2005) Endothelial t-PA release is impaired in overweight and obese adults but can be improved with regular aerobic exercise. Am J Physiol Endocrinol Metab 289: 807-813.

10. Kathiresan S, et al. (2006) Common genetic variation in five thrombosis genes and relations to plasma hemostatic protein level and cardiovascular disease risk. Arterioscler Thromb Vasc Biol 26:1405-1412.

11. Leadenhall P, et al. (2002) Tissue-type plasminogen activator $-7,351 \mathrm{C} / \mathrm{T}$ enhancer polymorphism is associated with a first myocardial infarction. Thromb Haemost 87: 105-109.

12. Stead NW, et al. (1983) Venous thrombosis in a family with the defective release of vascular plasminogen activator and elevated plasma factor VIII/von Willebrand's factor. Am J Med 74: 33-39.

13. Alessi MC, I Juhan-Vague (2004) Contribution of PAI-1 in cardiovascular pathology. Arch Mal Coeur Vaiss 97: 673-678. 14. Robinson SD, et al. (2007) Endothelial fibrinolytic capacity predicts future adverse cardiovascular events in patients with coronary heart disease. Arterioscler Thromb Vasc Biol 27: 1651-1656. 
15.

Thogersen AM, et al. (1998) High plasminogen activator inhibitor and tissue plasminogen activator levels in plasma precede a first acute myocardial infarction in both men and women: evidence for the fibrinolytic system as an independent primary risk factor. Circulation 98: 2241-2247.

16. Dunoyer-Geindre S, EK Kruithof (2011) Epigenetic control of tissue-type plasminogen activator synthesis in human endothelial cells. Cardiovasc Res 90: 457-463.

17. Larsson P, et al. (2013) Histone deacetylase inhibitors stimulate tissue-type plasminogen activator production in vascular endothelial cells. J Thromb Thrombolysis 35: 185-192.

18. Larsson P, et al. (2012) Role of histone acetylation in the stimulatory effect of valproic acid on vascular endothelial tissuetype plasminogen activator expression. PLoS One7: 31573.

19. Svennerholm K, et al. (2014) Histone deacetylase inhibitor treatment increases coronary t-PA release in a porcine ischemia model. PLoS One 9: 97260.

20. Larsson P, et al. (2016) Valproic acid selectively increases vascular endothelial tissue-type plasminogen activator production and reduces thrombus formation in the mouse. J Thromb Haemost 14: 2496-2508.

21. Saluveer O, et al. (2014) Profibrinolytic effect of the epigenetic modifier valproic acid in man. PLoS One 9: 107582.

22. Svennerholm K, et al. (2015) Histone deacetylase inhibition enhances tissue plasminogen activator release capacity in atherosclerotic man. PLoS One 10: 0121196.

23. Brookes RL, et al. (2018) Sodium Valproate, a Histone Deacetylase Inhibitor, Is Associated With Reduced Stroke Risk After Previous Ischemic Stroke or Transient Ischemic Attack. Stroke 49: 54-61.

24. Olesen JB, et al. (2011) Valproate attenuates the risk of myocardial infarction in patients with epilepsy: a nationwide cohort study. Pharmacoepidemiol Drug Saf 20: 146-53.

25. Dregan A, et al. (2014) Is sodium valproate, an HDAC inhibitor, associated with reduced risk of stroke and myocardial infarction? A nested case-control study. Pharmacoepidemiol Drug Saf 23: 759-67.

26. Olesen JB, et al. (2011) Effects of epilepsy and selected antiepileptic drugs on the risk of myocardial infarction, stroke, and death in patients with or without previous stroke: a nationwide cohort study. Pharmacoepidemiol Drug Saf 20: 964-971.

27. Loscher W (2002) Basic pharmacology of valproate: a review after 35 years of clinical use for the treatment of epilepsy. CNS Drugs 16: 669-94.

28. Cannon CP, et al. (1997) Circadian variation in the onset of unstable angina and non-Q-wave acute myocardial infarction (the TIMI III Registry and TIMI IIIB). Am J Cardiol 79:
29. Scheer FA, SA Shea (2014) Human circadian system causes a morning peak in prothrombotic plasminogen activator inhibitor-1 (PAI-1) independent of the sleep/wake cycle. Blood 123: 590-593.

30. European Pharmacopeia (2005) Disintegration of tablets and capsules.

31. Bostrom J, et al. (2013) Potent fibrinolysis inhibitor discovered by shape and electrostatic complementarity to the drug tranexamic acid. J Med Chem 56: 3273-2380.

32. Leppik IE, CA Hovinga (2013) Extended-release antiepileptic drugs: a comparison of pharmacokinetic parameters relative to original immediate-release formulations. Epilepsia 54: 28-35.

33. Eriksson H, et al. (2007) CPU inhibition with AZD9684: profibrinolytic effects in acute patients. J Thromb Haemost 5 Supplement 2: 367 .

34. Cieslik J, et al. (2018) Altered plasma clot properties increase the risk of recurrent deep vein thrombosis: a cohort study. Blood 131: 797-807.

35. Karasu A, et al. (2016) Prolonged clot lysis time increases the risk of a first but not recurrent venous thrombosis. Br J Haematol 172: 947-953.

36. Traby L, et al. (2012) Prediction of recurrent venous thromboembolism by clot lysis time: a prospective cohort study. PLoS One 7: 51447.

37. Undas A, (2017) Prothrombotic Fibrin Clot Phenotype in Patients with Deep Vein Thrombosis and Pulmonary Embolism: A New Risk Factor for Recurrence. Biomed Res Int 8196256.

38. Undas A, M Zabczyk (2018) Antithrombotic medications and their impact on fibrin clot structure and function. J Physiol Pharmacol 69.

39. Zabczyk M, J Majewski, J Lelakowski, ( $\left.\begin{array}{llll}2 & 0 & 1 & 1\end{array}\right)$ Thromboembolic events are associated with prolonged clot lysis time in patients with permanent atrial fibrillation. Pol Arch Med Wewn 121: 400-407.

40. Sumaya W, et al. (2018) Fibrin clot properties independently predict adverse clinical outcome following acute coronary syndrome: a PLATO substudy. Eur Heart J 39: 1078-1085.

41. Ajjan RA, et al. (2009) Effects of aspirin on clot structure and fibrinolysis using a novel in vitro cellular system. Arterioscler Thromb Vasc Biol 29: 712-717.

42. Lau YC, et al. (2016) Effects of non-vitamin K antagonist oral anticoagulants on fibrin clot and whole blood clot formation, integrity and thrombolysis in patients with atrial fibrillation. J Thromb Thrombolysis 42: 535-544.

253-258. 
43. (2018) National Institue for Health and Care Excellence. British National Formulary.

44. Bishton MJ, et al. (2011) Deciphering the molecular and biologic processes that mediate histone deacetylase inhibitor-induced thrombocytopenia. Blood 117: 3658-3668.

45. Nasreddine WA (2008) Beydoun, Valproate-induced thrombocytopenia: a prospective monotherapy study. Epilepsia 49: 438-445.

46. Koenig S, et al. (2008) High incidence of vaproate-induced coagulation disorders in children receiving valproic acid: a prospective study. Blood Coagul Fibrinolysis 19: 375-382.

Submit your manuscript to a JScholar journal and benefit from:

q Convenient online submission

ฯ Rigorous peer review

I Immediate publication on acceptance

ब Open access: articles freely available online

q High visibility within the field

I Better discount for your subsequent articles

Submit your manuscript at http://www.jscholaronline.org/submit-manuscript.php 